\title{
Ultra-high field MR angiography in human migraine models: a 3.0 T/7.0 T comparison study
}

\author{
Casper Emil Christensen ${ }^{1}$, Samaira Younis ${ }^{1}$, Ulrich Lindberg ${ }^{2}$, Vincent Oltman Boer ${ }^{3}$, Patrick de Koning ${ }^{4}$, \\ Esben Thade Petersen ${ }^{3,5}$, Olaf Bjarne Paulson ${ }^{6}$, Henrik Bo Wiberg Larsson ${ }^{2}$, Faisal Mohammad Amin ${ }^{1 \dagger}$ and \\ Messoud Ashina ${ }^{1 *+}$
}

\begin{abstract}
Background: Sildenafil and calcitonin gene-related peptide both dilate the intradural segments of the middle meningeal artery measured with 3.0 tesla (T) MR angiography. Here we hypothesized that an increase in field strength to 7.0 T and concomitant enhanced voxel resolution would lower variance in measurements of dilation in the intradural middle meningeal artery.

Methods: Five subjects completed two sessions at respectively 3.0 T and 7.0 T. Each session comprised MR angiography scans once before and twice after administration of sildenafil, calcitonin gene-related peptide or placebo in a three-way, crossover, double-blind, placebo-controlled design.

Results: Standard deviations of arterial circumference revealed no difference between 3.0 T and 7.0 T measurements $(p=0.379)$. We found a decrease in standard deviation from our original angiography analysis software (QMra) to a newer (LAVA) software package $(p<0.001)$. Furthermore, we found that the dilation after sildenafil and calcitonin gene-related peptide were comparable between 3.0 T and 7.0 T.
\end{abstract}

Conclusions: Our findings suggest no gain from the increase in voxel resolution but cemented dilatory findings from earlier. The implemented software update improved variance in circumference measurements in the intradural middle meningeal artery, which should be exploited in future studies.

Trial registration: The study is part of a parent study, which is registered at ClinicalTrials.gov (NCT03143465).

Keywords: Middle meningeal artery, Dura mater, Neurovascular, Sildenafil, Calcitonin gene-related peptide

\section{Background}

Neurovascular mechanisms of migraine headache has been studied extensively using magnetic resonance angiography (MRA) [1-5]. The middle meningeal artery (MMA) is an essential focus of these studies, both in search for the origin of pain and as a physiological measure of altered neurovascular modulation [6]. Two MRA studies found the extracranial part of MMA to be dilated more on the pain side than non-pain side in migraine

\footnotetext{
* Correspondence: ashina@dadlnet.dk

${ }^{\dagger}$ Faisal Mohammad Amin and Messoud Ashina contributed equally to this work.

${ }^{1}$ Danish Headache Center and Department of Neurology, Rigshospitalet Glostrup, Faculty of Health and Medical Sciences, University of Copenhagen, Valdemar Hansens Vej 5, 2600 Glostrup, Denmark

Full list of author information is available at the end of the article
}

patients during provoked attacks in human migraine models [1, 4]. If MMA dilation reflects activation of trigeminal nociceptors and meningeal neuropeptide release, then the intracranial segments of MMA might represent more local changes in the dural environment than the extracranial part.

In an effort to study dilation in the intracranial segments of the MMA, we recently performed a 3.0 tesla (T) MRA study in healthy volunteers showing dilation of the intradural MMA after administration of headache-inducing substances calcitonin gene-related peptide (CGRP) and sildenafil [7]. Changes in MMA dimensions within subjects over time in previous MRA studies vary from 30\% [3] to as little as $\sim 5 \%$ [4] which challenges detection rate and power in costly and time-consuming MRI studies. 
Furthermore, the route of the MMA in the cranial convexity potentially limits the cross-sectional MRA resolution [8].

In this study, we wanted to investigate whether the uncertainty - defined as standard deviation (SD) of measurements along the course of the intradural MMA improves when increasing the field strength from $3.0 \mathrm{~T}$ to $7.0 \mathrm{~T}$, potentially increasing spatial resolution and diminishing the partial volume effect. We used a randomized, placebo-controlled, three-way, crossover, double-dummy setup at $7.0 \mathrm{~T}$ in participants who had previously gone through the exact same setup at $3.0 \mathrm{~T}$ and we compared standard deviations in measurements between the two systems.

\section{Methods}

\section{Participants and approvals}

We recruited healthy volunteers through a Danish recruitment website (www.forsoegsperson.dk). Participants were offered enrollment in this $7.0 \mathrm{~T}$ continuation study upon completion of the prior $3.0 \mathrm{~T}$ study, results of which have been published elsewhere [7]. Both men and women were eligible for inclusion if they were between 18 and 50 years of age, weighed 50 to $100 \mathrm{~kg}$ and had successfully completed the $3.0 \mathrm{~T}$ part of the parent study. Exclusion criteria were a history of somatic and/ or psychiatric disease any primary headache disorder, other than infrequent episodes of tension type headache (less than 2 days/month), having first-degree relatives with migraine, being pregnant or breastfeeding, use of daily medication, other than oral contraceptives and not using safe contraceptive methods. Furthermore, volunteers were excluded if there were contraindications for MRI scans (i.e. ferromagnetic implants, recent surgical procedures, claustrophobia, etc.) or any other condition deemed by the investigator to be incompatible with participation. Participants provided written informed consent in accordance with the declaration of Helsinki and the study was approved by the Ethics committee of the Capital Region of Denmark (H-15019063) along with the Danish Medicines Agency (CIV-16-12-017964). The study is part of a parent study, which is registered at ClinicalTrials.gov (NCT03143465) and other parts of the study have been or will be published elsewhere.

\section{Study design}

Participants were allocated to receive CGRP, sildenafil and placebo in random order on three separate study days. Drug administration was preceded by a 7.0 T MRA and succeeded by two additional scans at $30 \mathrm{~min}$ and 120 min post-administration. On each day, participants would receive an i.v. infusion of isotonic saline or CGRP as well as an oral administration of $100 \mathrm{mg}$ sildenafil or placebo, where the combination was dependent on randomization (placebo+CGRP/sildenafil+placebo/placebo+placebo). This design was a replica of the previous 3.0 T study which all participants had previously completed at Rigshospitalet Glostrup, resulting in paired observations between $3.0 \mathrm{~T}$ and $7.0 \mathrm{~T}$.

\section{Experimental procedures}

Participants arrived at the Danish Research Centre for Magnetic Resonance headache-free on the morning of each study day. They were required to avoid intake of any medication (other than oral contraceptives), coffee, tea, cocoa, other caffeinated beverages and methylxanthinecontaining food $12 \mathrm{~h}$ before the first scan and to fast $4 \mathrm{~h}$ before commencing the experiment. After arrival, a peripheral venous catheter was placed in a cubital vein and participants were placed in the scanner. They were instructed to stay awake during the recordings.

MR angiography scans were obtained at baseline $\left(\mathrm{T}_{\mathrm{Ba}-}\right.$ seline) followed by drug administration at $\mathrm{T}_{0}$ and two additional MRI scans at $T_{30}$ and $T_{120}$. Participants were monitored with an Expression In Vivo monitor (Philips Medical, Orlando, FL, USA) continuously, regarding blood pressure, heart rate, end-tidal $\mathrm{CO}_{2}$, respiratory rate and pulse oximetry every $10 \mathrm{~min}$ following drug administration. A standardized headache questionnaire was used to evaluate any headache-related symptoms for the duration of the study where pain was scored on a numerical rating scale (NRS) from 0 (no pain) to 10 (maximum imaginable pain).

\section{Data acquisition}

The acquisition of the $3.0 \mathrm{~T}$ scans used for comparisons in this study are described in detail elsewhere [7]. The $3.0 \mathrm{~T}$ MRI scans were performed on a Philips Achieva dStream Scanner (Philips Medical Systems, Netherlands) using a 32-channel phased-array head coil. A three-dimensional time-of-flight (TOF) MRA was conducted with the following parameters:

Field of view $200 \times 200 \times 36.75 \mathrm{~mm}^{3}$, acquired matrix size $800 \times 570$, acquired voxel resolution $0.25 \times 0.35 \times$ $0.70 \mathrm{~mm}^{3}$, reconstructed voxel resolution $0.20 \times 0.20 \times$ $0.35 \mathrm{~mm}^{3}$, TR 23 milliseconds, TE 3.5 milliseconds, flip angle $18^{\circ}$, SENSE p reduction $2.5,3$ chunks, duration 13 $\min 06 \mathrm{~s}$.

The 7.0 T MRI scans were performed on a 7.0 T Philips Achieva Scanner (Philips Medical Systems, Netherlands) using a two-channel volume transmit head coil with 32-channel receiver array (Nova Medical, Inc., Burlington, MA, US). Again, a three-dimensional TOF MRA sequence was used with the following parameters:

Field of view $200 \times 190.4 \times 32.4 \mathrm{~mm}^{3}$, acquired matrix size $668 \times 636$, acquired voxel resolution $0.3 \times 0.3 \times 0.3$ $\mathrm{mm}^{3}$, reconstructed voxel resolution $0.15 \times 0.15 \times 0.15$ $\mathrm{mm}^{3}$, TR 19 milliseconds, TE 2.6 milliseconds, flip angle 
$30^{\circ}$, SENSE p reduction $4.5,3$ chunks, duration 9 min $58 \mathrm{~s}$.

High-dielectric permittivity pads were placed over the temporomandibular joint to improve image quality at the base of the skull, where intradural segments of the MMA is located [9].

\section{Data analysis}

MR angiography data was transferred to a separate work station in the DICOM format and analyzed using LKEB QMra, and the updated LKEB LAVA vessel contour detection software $[10,11]$. The software allows the user to pinpoint start- and endpoint of vessels for contour analysis, propagates a center line through the vessel and provides circumference slices perpendicular to this line along the chosen segment. If slices were distorted, noisy or otherwise immeasurable, they were discarded. The user made sure to select the same segments within each subject between scans to provide reliable measurements of change over time. We analyzed the intradural segment of MMA, identified as the innermost part covered by the field of view, approximately $20 \mathrm{~mm}$ after MMA enters the skull through the foramen spinosum. Ultimately, a $5 \mathrm{~mm}$ segment is chosen comprising up to 34 slices and the mean and standard deviation is computed for that segment at that time point. 7.0 T MRA resolution required us to use updated contour detection software (LAVA), which was not used in the original study, causing us to reanalyze the previous data using the new software post hoc. To further explore the potential effect of the resolution on $7.0 \mathrm{~T}$, we resampled the $7.0 \mathrm{~T}$ data, decreasing the resolution, to mimic the original $3.0 \mathrm{~T}$ scans. Both QMra and LAVA analyses are reported, and the software change was factored into analyses (see below). All analyses of $3.0 \mathrm{~T}$ and $7.0 \mathrm{~T}$ scans were performed on the same workstation by CEC.

\section{Statistical analyses}

Our primary endpoint was difference in SDs of vessel segment measurements in intradural MMA at $3.0 \mathrm{~T}$ vs. 7.0 T within subject, within drug, within time point providing us with a maximum of $5_{\text {subjects }} \times 3_{\text {days }} \times 3_{\text {times }} \times$ $2_{\text {sides }}=90$ paired observations. Vessel segment standard deviation was analyzed with a linear mixed effects model with scanner and contour detection software as fixed effects and subject, drug and time point as random effects. Secondary analyses on drug effect on circumference over time were performed descriptively as percentage change from baseline and using Bland-Altman agreement statistics between scanners [12].

All analyses were performed using $\mathrm{R}$ (version 3.4.2) with packages lme4 (version 1.1-17) and BlandAltmannLeh (version 0.3.1), $p$-values are reported as two-tailed with a significance level of $5 \%$.

\section{Results}

Five participants who had completed the $3.0 \mathrm{~T}$ parent study went on to complete the $7.0 \mathrm{~T}$ extension. Two men and three women mean age 24 (range 20 to 29). The median number of days between $3.0 \mathrm{~T}$ and $7.0 \mathrm{~T}$ studies were 476 (range 330 to 501).

Mean SD for intradural MMA on 3.0 T with QMra software was $0.332 \mathrm{~mm}$ (SE 0.033), mean circumference was $4.18 \mathrm{~mm}$ (95\% CI [4.04 to 4.32]). We found no difference

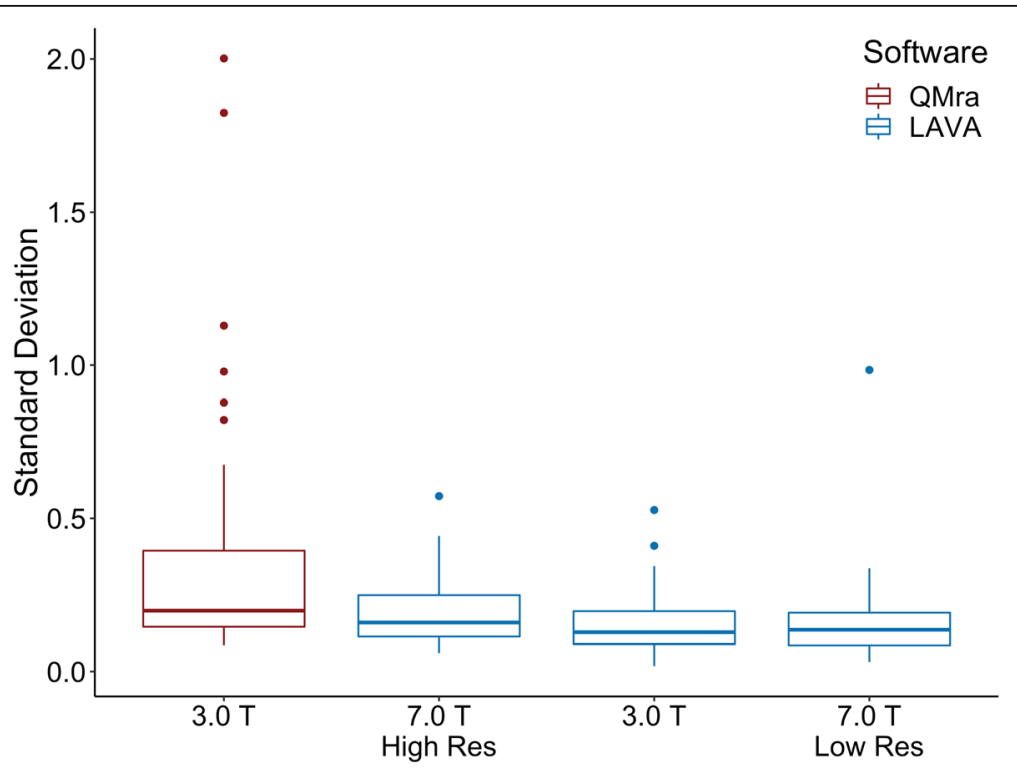

Fig. 1 Standard deviations of the intradural MMA measurements in the four analysis iterations. 7.0 T High Res: 7.0 T scan at full resolution; 7.0 T Low Res: 7.0 T scan resampled to 3.0 T resolution 


\section{$3.0 \mathrm{~T}$}

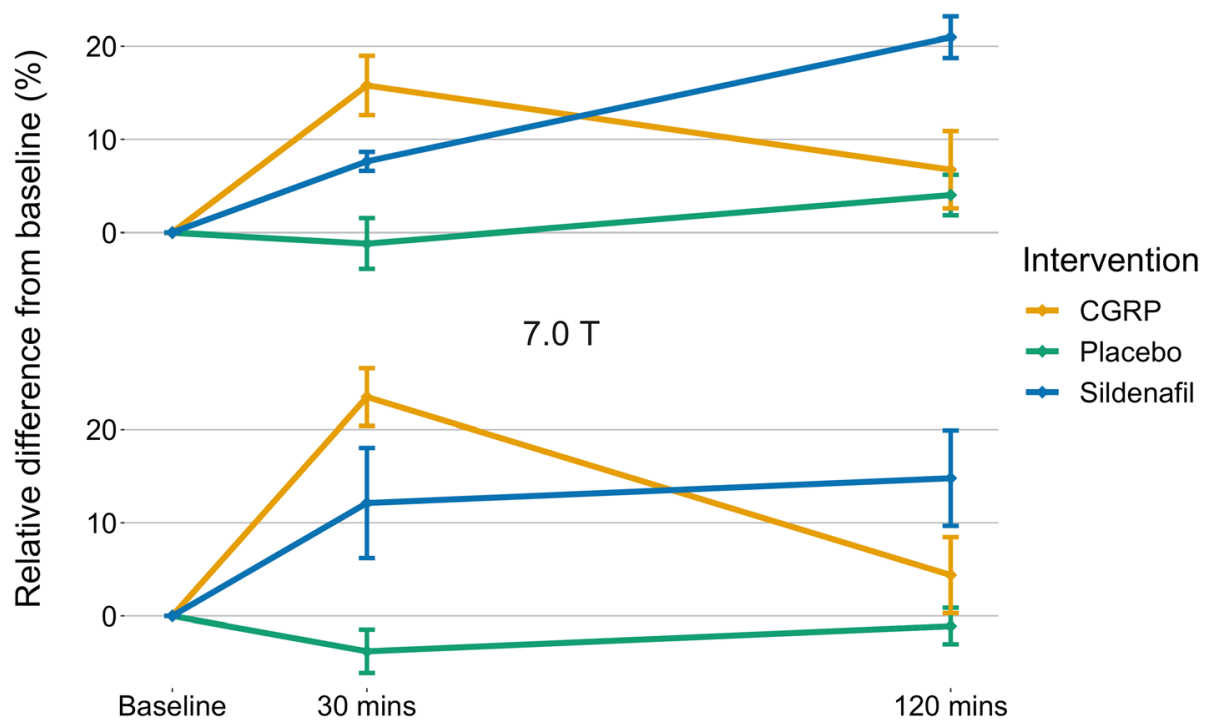

Fig. 2 Change in intradural MMA circumference over time after each of the three interventions. 3.0 T and 7.0 T data is depicted as mean \pm SE, all data analyzed with LAVA software

in SD between $3.0 \mathrm{~T}$ and $7.0 \mathrm{~T}$ when applying the same LAVA software on both $(p=0.379)$, but SD was $0.184 \mathrm{~mm}$ lower for LAVA than for QMra independent of scanner field strength $(p<0.001)$. We found no difference between resampled and original 7.0 T standard deviations (Fig. 1).

Arterial circumference change over time between the two scanners are shown in Fig. 2. We found similar patterns across the three interventions with immediate increase in arterial circumference both after CGRP $(15.8 \%$ at $3.0 \mathrm{~T}$ and $23.5 \%$ at $7.0 \mathrm{~T})$ and sildenafil $(7.6 \%$ at $3.0 \mathrm{~T}$ and $12.1 \%$ at $7.0 \mathrm{~T})$. Dilation after sildenafil appears to be sustained after $120 \mathrm{~min}(21.0 \%$ at $3.0 \mathrm{~T}$ and
$14.8 \%$ at $7.0 \mathrm{~T}$ ) contrary to the CGRP-induced dilation (Fig. 2). Correspondingly, Fig. 3 shows relatively high levels of agreement between circumference measurements at 3.0 $\mathrm{T}$ and 7.0 $\mathrm{T}$ within subject, drug and time (Fig. 3).

Examples of the different angiography recordings are shown in Fig. 4. We observed an apparent decrease in mean arterial pressure after sildenafil (Fig. 5) and a small increase in heart rate after CGRP (not shown).

\section{Discussion}

The main outcome of this study was that we found no difference in standard deviation of measurements of

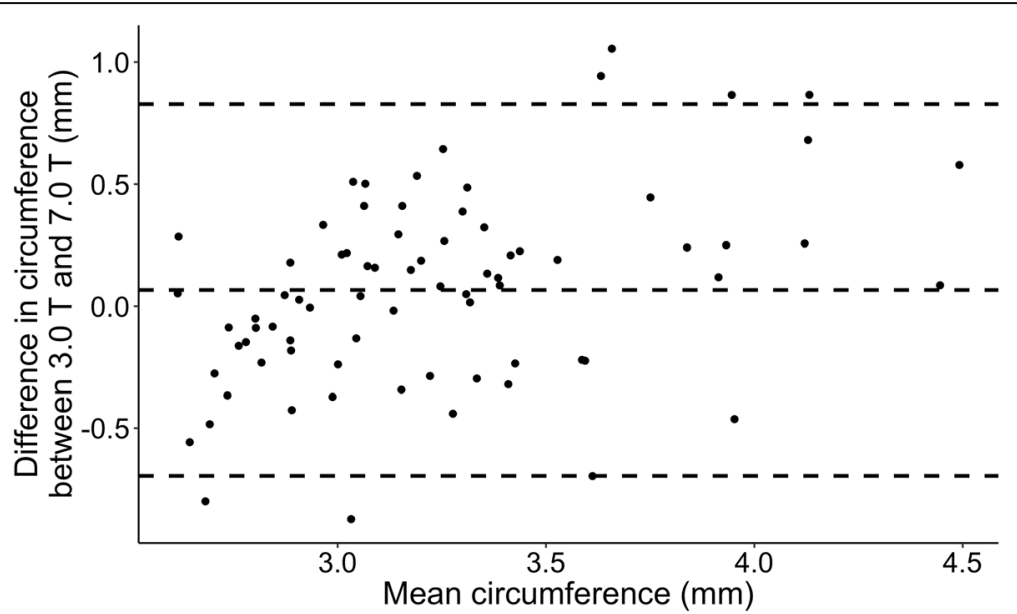

Fig. 3 Bland-Altmann plot depicting agreement between circumference measurements of intradural MMA 3.0 T vs 7.0 T within subject, within drug, within time point analyzed with LAVA software. Each point represents difference in circumference between $3.0 \mathrm{~T}$ and $7.0 \mathrm{~T}$ as a function of mean of the two measurements. Dashed lines are overall mean of differences $\pm 2 S D$ 


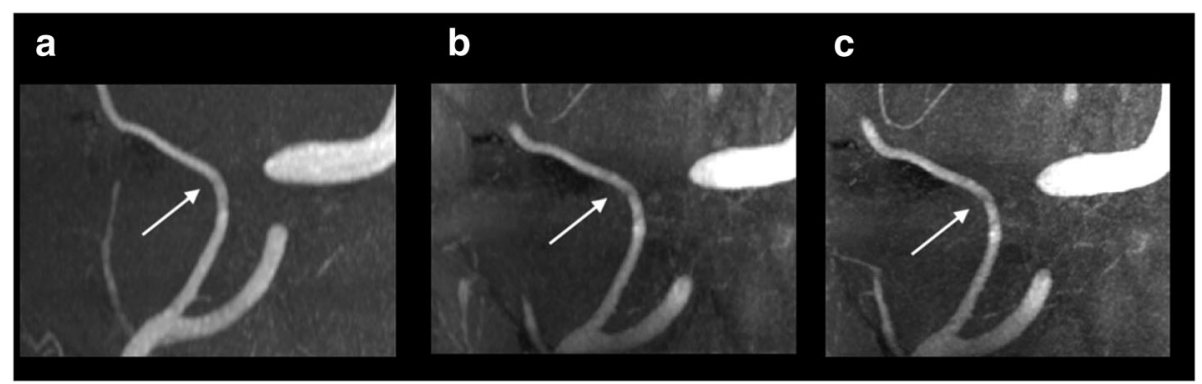

Fig. 4 Examples of the middle meningeal artery (arrow) in three different data sets. a: 3.0 T scan; b: 7.0 T scan at low resolution; c: 7.0 T scan at high resolution

intradural MMA circumference between $3.0 \mathrm{~T}$ and 7.0 T. We did, however, uncover a difference in standard deviation between the previously used QMra circumference detection software and the newer LAVA. Furthermore, we showed high robustness in measurements of vascular effects of CGRP and sildenafil over time as the temporal evolution from drug administration was similar between the 3.0 and $7.0 \mathrm{~T}$ scan days (Fig. 2).

Sildenafil is a selective phosphodiesterase- 5 inhibitor that impedes degradation of cyclic guanosine monophosphate (cGMP) which ultimately leads to relaxation of vascular smooth muscle cells. [13] Calcitonin gene-related peptide relaxes vascular smooth muscle via a G-protein coupled receptor-mediated increase in cyclic adenosine monophosphate (cAMP) [14]. We have previously shown the ability to measure intradural MMA circumference increase after either sildenafil or CGRP using high resolution $3.0 \mathrm{~T}$ MRA [7]. The intradural branches of the MMA move in all three planes and we hypothesized, that we could improve confidence in our caliber measurements, if we streamlined all dimensions of the voxel size on MRA. In our previous study, the lowest cross-sectional resolution was $0.35 \times 0.70 \mathrm{~mm}^{2}$, whereas at
7.0 T, we could increase the resolution to $0.3 \times 0.3 \mathrm{~mm}^{2}$. However, this increase in resolution did not change the standard deviations along the vessel segment measurements. Neither did the temporal evolution of intradural MMA caliber change, when moving to $7.0 \mathrm{~T}$, showing absolute robustness of our measurements from $3.0 \mathrm{~T}$ and consolidating our previous findings (Figs. 2 and 3).

The contour detection software works by detecting a pathline of the vessel chosen by the operator. Then a model is fitted to the underlying image data defining a 3D mesh in non-uniform rational basis spline fashion [11]. QMra software moves along the pathline and determines the best candidate in neighboring voxels as next in line in the vessel contour [10]. The newer LAVA program scans a wider range of potential voxels and locates the best candidates using the maximum gradient [11]. The older QMra software is thus more susceptible to poor pathline estimations and noise whereas LAVA looks at a broader range of voxels and is more resilient to these effects.

In MRA research in migraine, changes in dimensions of the MMA have been proposed a marker of meningeal activation during migraine attacks, and changes reported

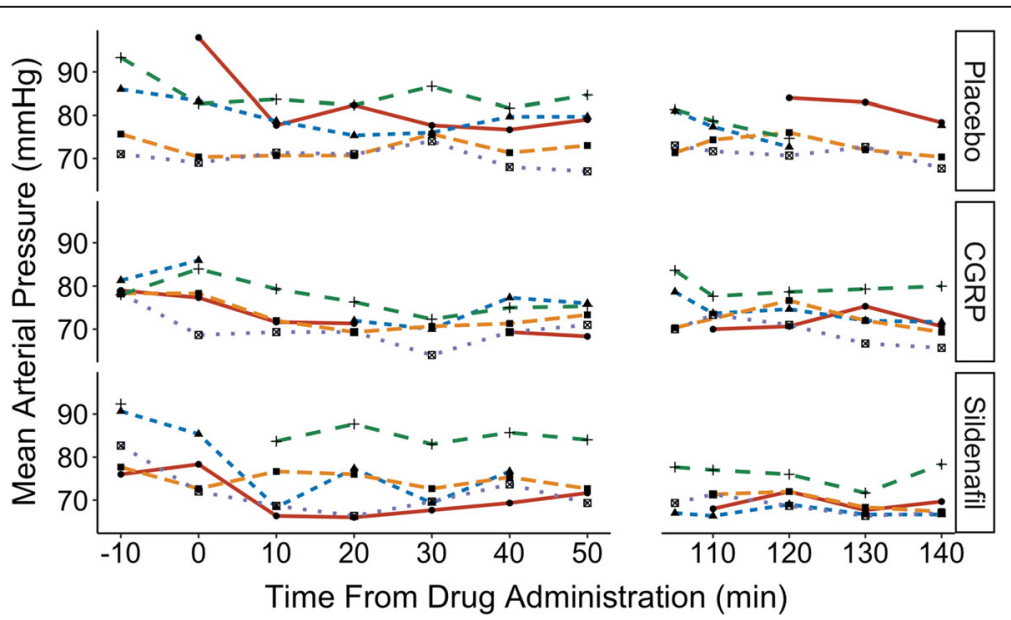

Fig. 5 Mean arterial blood pressure by time in the individual participants after each of the three interventions 
are as small as $\sim 5 \%$ corresponding to around $0.15 \mathrm{~mm}$ in the intradural MMA circumference [4]. Needless to say, a high level of confidence in measurements is needed, and here we show robust MMA measurements from our 3.0 T results even when re-testing at ultra-high field strength.

\section{Strengths and limitations}

We tested standard deviations along vessel segments in randomized, double-blind, placebo-controlled fashion using the same subjects on the two different systems resulting in quite strong paired comparisons. Furthermore, contour detection is a semi-automated procedure with minimal operator influence [15]. A central limitation is the time between $3.0 \mathrm{~T}$ and $7.0 \mathrm{~T}$ scans with a median of 476 days between them.

\section{Conclusions}

We found no reduction in standard deviation with the increase in voxel resolution from $3.0 \mathrm{~T}$ to $7.0 \mathrm{~T}$. The implemented software update, however, improved variance in circumference measurements in the intradural MMA, which should be exploited in future studies. Furthermore, we affirmed the dilatory response in intradural MMA after sildenafil and CGRP that was reported previously [7].

\section{Abbreviations \\ CAMP: cyclic Adenosine Monophosphate; cGMP: cyclic Guanosine Monophosphate; CGRP: Calcitonin Gene-Related Peptide; MMA: Middle Meningeal Artery; MRA: Magnetic Resonance Angiography; NRS: Numeric Rating Scale; SD: Standard Deviation; T: Tesla; TOF: Time-Of-Flight}

\section{Acknowledgements}

The authors thank the participants for their contribution, Drs. Hashmat Ghanizada and Anne Luise Vollesen for assistance with drug preparation and randomization, as well as the section of biostatistics at University of Copenhagen for assistance with data analyses.

\section{Funding}

This study was supported by the Lundbeck foundation (R155-2014-171 and R249-2017-1608) as well as the A. P Moeller foundation (17-L-0384). The national 7 T MRI scanner was funded by the Danish Agency for Science, Technology and Innovation grant no. 0601-01370B, and The John and Birthe Meyer Foundation. None of the funding bodies had any influence on study design, inclusion of participants, collection or interpretation of data.

\section{Availability of data and materials}

Data from this study are available from the corresponding author upon reasonable request.

\section{Authors' contributions}

CEC: Study concept and design, acquisition, analysis, and interpretation of data, and drafting and revision of manuscript. SY: Study concept and design, acquisition of data and revision of manuscript. ULNOB/ETP: Study concept and design, development of MRI protocol, interpretation of data and revision of manuscript. PDK: Development and support regarding processing software, interpretation of data and revision of manuscript. OBP/HBWL: Study concept and design, interpretation of data, revision of manuscript. FMA/MA: Study concept and design, interpretation of data, revision of manuscript and overall supervision of study. All authors read and approved the final manuscript.

\section{Ethics approval and consent to participate}

All participants provided written informed consent in accordance with the declaration of Helsinki and the study was approved by the Ethics committee of the capital region of Denmark $(\mathrm{H}-15019063)$ along with the Danish Medicines Agency (CIV-16-12-017964).

\section{Consent for publication}

Not Applicable

\section{Competing interests}

CEC/SY/UL/NOB/PDK/ETP/OBP/HBWL report no conflicts of interest pertaining to this work. FMA has received personal fees and/or honoraria for lecturing from Teva, Eli Lilly and Novartis. FMA is principal investigator for a Novartis Phase IV trial and member of advisory boards for Eli Lilly and Novartis.

MA is a consultant or scientific advisor for Allergan, Amgen, Alder, Eli Lilly, Novartis and Teva, principal investigator for Amgen 20120178 (Phase II), 20120295 (Phase II), 20130255 (Open label extension), 20120297 (Phase III), 20150308 (Phase II), ElectroCore GM-11 gamma-Core-R, TEVA TV48125-CNS30068 (Phase III), Novartis CAMG334A2301 (Phase III) and Alder PROMISE-2 (Phase III). MA has no ownership interest and does not hold stock in any pharmaceutical company. MA serves as associated editor of Cephalalgia and co-editor of the Journal of Headache and Pain.

\section{Publisher's Note}

Springer Nature remains neutral with regard to jurisdictional claims in published maps and institutional affiliations.

\section{Author details}

${ }^{1}$ Danish Headache Center and Department of Neurology, Rigshospitalet Glostrup, Faculty of Health and Medical Sciences, University of Copenhagen, Valdemar Hansens Vej 5, 2600 Glostrup, Denmark. ²Functional Imaging Unit, Department of Clinical Physiology, Nuclear Medicine and PET, Rigshospitalet, Faculty of Health and Medical Sciences, University of Copenhagen,

Copenhagen, Denmark. ${ }^{3}$ Danish Research Centre for Magnetic Resonance, Centre for Functional and Diagnostic Imaging and Research, Amager and Hvidovre Hospital, Copenhagen, Denmark. ${ }^{4}$ Division of Image Processing, Department of Radiology, Leiden University Medical Center, Leiden, Netherlands. ${ }^{5}$ Center for Magnetic Resonance, Department of Health Technology, Technical University of Denmark, Kgs Lyngby, Denmark. ${ }^{6}$ Neurobiology Research Unit, Department of Neurology, Rigshospitalet Blegdamsvej, Faculty of Health and Medical Sciences, University of Copenhagen, Copenhagen, Denmark.

Received: 15 February 2019 Accepted: 16 April 2019

Published online: 06 May 2019

\section{References}

1. Asghar MS, Hansen AE, Amin FM et al (2011) Evidence for a vascular factor in migraine. Ann Neurol 69:635-645

2. Amin FM, Asghar MS, Hougaard A et al (2013) Magnetic resonance angiography of intracranial and extracranial arteries in patients with spontaneous migraine without aura: a cross-sectional study. Lancet Neurol 12:454-461

3. Amin FM, Hougaard A, Schytz HW et al (2014) Investigation of the pathophysiological mechanisms of migraine attacks induced by pituitary adenylate cyclase-activating polypeptide-38. Brain 137:779-794

4. Khan S, Amin FM, Christensen CE et al (2019) Meningeal contribution to migraine pain: a magnetic resonance angiography study. Brain 142:93-102

5. Arngrim N, Schytz HW, Britze J et al (2016) Migraine induced by hypoxia: an MRI spectroscopy and angiography study. Brain 139:723-737

6. Olesen J, Burstein R, Ashina M, Tfelt-Hansen P (2009) Origin of pain in migraine: evidence for peripheral sensitisation. Lancet Neurol 8:679-690

7. Christensen CE, Amin FM, Younis S et al (2019) Sildenafil and calcitonin gene-related peptide dilate intradural arteries: a 3T MR angiography study in healthy volunteers. Cephalalgia 39:264-273

8. Kornieieva M, Hadidy A, Zhuravlova I (2015) Variability of the middle meningeal artery subject to the shape of skull. J Neurol Surg B Skull Base 76:451-458 
9. Snaar JEM, Teeuwisse WM, Versluis MJ et al (2011) Improvements in highfield localized MRS of the medial temporal lobe in humans using new deformable high-dielectric materials. NMR Biomed 24:873-879

10. de Koning PJH, Schaap JA, Janssen JP, Westenberg JJM, van der Geest RJ, Reiber JHC (2003) Automated segmentation and analysis of vascular structures in magnetic resonance angiographic images. Magn Reson Med 50:1189-1198

11. van 't Klooster R, de Koning PJH, Dehnavi RA et al (2012) Automatic lumen and outer wall segmentation of the carotid artery using deformable threedimensional models in MR angiography and vessel wall images. J Magn Reson Imaging 35:156-165

12. Martin Bland J, Altman D (1986) Statistical methods for assessing agreement between two methods of CLINICAL MEASUREMENT. Lancet 327:307-310

13. Jackson G, Benjamin N, Jackson N, Allen MJ (1999) Effects of sildenafil citrate on human hemodynamics. Am J Cardiol 83:13-20

14. Brain SD, Grant AD (2004) Vascular actions of calcitonin gene-related peptide and Adrenomedullin. Physiol Rev 84:903-934

15. Amin FM, Lundholm E, Hougaard A et al (2014) Measurement precision and biological variation of cranial arteries using automated analysis of $3 \mathrm{~T}$ magnetic resonance angiography. J Headache Pain 15:25

Ready to submit your research? Choose BMC and benefit from:

- fast, convenient online submission

- thorough peer review by experienced researchers in your field

- rapid publication on acceptance

- support for research data, including large and complex data types

- gold Open Access which fosters wider collaboration and increased citations

- maximum visibility for your research: over $100 \mathrm{M}$ website views per year

At $\mathrm{BMC}$, research is always in progress.

Learn more biomedcentral.com/submissions 